\title{
Calculating Infinite-Medium $\alpha$-Eigenvalue Spectra with Monte Carlo using a Transition Rate Matrix Method ${ }^{\text {in }}$
}

\author{
Benjamin R. Betzler ${ }^{\mathrm{a}, 1, *}$, Brian C. Kiedrowski ${ }^{\mathrm{a}}$, Forrest B. Brown ${ }^{\mathrm{b}}$, William \\ R. Martin ${ }^{\mathrm{a}}$ \\ ${ }^{a}$ Department of Nuclear Engineering and Radiological Sciences, University of Michigan, \\ 2355 Bonisteel Boulevard, Ann Arbor, MI 48109, USA \\ ${ }^{b}$ Los Alamos National Laboratory, P.O. Box 1663, MS A143 Los Alamos, NM 87545, \\ USA
}

\section{Abstract}

The time-dependent behavior of the energy spectrum in neutron transport was investigated with a formulation, based on continuous-time Markov processes, for computing $\alpha$ eigenvalues and eigenvectors in an infinite medium. For this, a research Monte Carlo code called "TORTE” (To Obtain Real Time Eigenvalues) was created and used to estimate elements of a transition rate matrix. TORTE is capable of using both multigroup and continuous-energy nuclear data, and verification was performed. Eigenvalue spectra for infinite

\footnotetext{
This manuscript has been authored by UT-Battelle, LLC under Contract No. DEAC05-00OR22725 with the U.S. Department of Energy. The United States Government retains and the publisher, by accepting the article for publication, acknowledges that the United States Government retains a non-exclusive, paid-up, irrevocable, world-wide license to publish or reproduce the published form of this manuscript, or allow others to do so, for United States Government purposes. The Department of Energy will provide public access to these results of federally sponsored research in accordance with the DOE Public Access Plan (http://energy.gov/downloads/doe-public-access-plan).

${ }^{*}$ Corresponding author

Email addresses: betzlerbr@ornl.gov (Benjamin R. Betzler), bckiedro@umich.edu (Brian C. Kiedrowski), fbrown@lanl.gov (Forrest B. Brown), wrm@umich.edu (William R. Martin)

${ }^{1}$ Present address: Oak Ridge National Laboratory, P.O. Box 2008, 1 Bethel Valley Road, Oak Ridge, TN 37831, USA
} 
homogeneous mixtures were obtained, and an eigenfunction expansion was used to investigate transient behavior of the neutron energy spectrum.

Keywords: Monte Carlo, time eigenvalue, Markov process, criticality

\section{Introduction}

A tally of the fission matrix may be used to estimate $k$ eigenvalues and eigenvectors of a system via Monte Carlo techniques (Carney et al., 2012). The premise is that the spatial domain is discretized and transition probabilities (probabilities of of particles born in cell $i$ causing a fission in cell j) are estimated by Monte Carlo tallies. For an adequately chosen spatial mesh, the eigenvalues and eigenvectors of the fission matrix match those of the underlying system.

The fission matrix formulation is a discrete-time Markov process where the time intervals represent fission generations. A more accurate description of temporal behavior is obtained when the fission matrix is extended to describe transitions in continuous time. This extension models the evolution of a system as a continuous-time Markov process, where the analog to the fission matrix is the transition rate matrix. A forward Monte Carlo simulation can be used to estimate elements of the transition rate matrix, similar to the way the fission matrix is estimated. The transition rate matrix can be used to define a master equation for the process, which is the Kolmogorov backward equation (Grimmett and Stirzaker, 2001). In the language of neutron transport, the eigenvalues of the transition rate matrix are the time-absorption or $\alpha$ eigenvalues, and the eigenvectors are discrete approximations of the eigenfunctions of the adjoint (backwards) transport 
equations. Current Monte Carlo methods for determining these $\alpha$ eigenvalues are limited: they are based on a computationally expensive $k$ - $\alpha$ iteration and are only able to obtain a few $\alpha$ eigenvalues (Hill, 1983; Yamamoto, 2011).

For this paper, the discrete states are defined to be not over the spatial domain, but intervals in the energy spectrum - the geometry is assumed to be homogeneous and infinite in extent. The tallies needed to estimate elements of the transition rate matrix are defined. A standalone code called "TORTE" (To Obtain Real Time Eigenvalues), capable of using either multigroup or continuous-energy nuclear data, was written in MATLAB (MATLAB, 2010), and verification results for multigroup systems are presented. The results of this verification show that, at least for infinite-medium multigroup problems, the transition rate matrix can accurately predict $\alpha$ eigenvalues. Observations of continuous-energy $\alpha$ eigenvalue spectra are given, and using an eigenfunction expansion, an approximation of the time-dependent behavior of the neutron energy spectrum is given. The results strongly suggest that the transition rate matrix is capable of producing accurate $\alpha$-eigenvalue spectra for continuous energy as well. This provides a basis for an extension of the transition rate matrix to consider geometry (space and angle) to study more realistic problems.

\section{Theory}

The Monte Carlo method performs direct simulation of neutron transport in systems. Through the use of mean values of estimators, called tallies, solutions to the neutron transport equation (which also describe mean-value neutron behavior) can be inferred. A typical assumption to handle time 
dependence is to assume that the temporal dimension is separable from the rest of phase space (position, energy, direction) and that the flux $\psi$ at any time $t$ can be described as

$$
\psi(\mathbf{r}, E, \hat{\mathbf{\Omega}}, t)=\sum_{n=1}^{\infty} A_{n} \psi_{n}(\mathbf{r}, E, \hat{\mathbf{\Omega}}) e^{\alpha_{n} t},
$$

where $\alpha_{n}$ is the $n$-th eigenvalue, $\psi_{n}$ is the corresponding shape eigenfunction, and $A_{n}$ is an amplitude coefficient related to the neutron source. Typically of most interest is $\alpha_{0}$, the largest or fundamental eigenvalue describing the asymptotic behavior of the system. In addition to separability, the completeness of the eigenfunctions is assumed. This has never been rigorously proven for a general system but seems to work well empirically (Larsen and Zweifel, 1974). For a complete description in a nuclear system with fission, delayed neutron precursor rate equations must also be included. The delayed neutron precursor concentrations $C_{m}(\mathbf{r}, t)$ also follow exponential time behavior.

\subsection{Transition rate matrix}

The $\alpha$ eigenvalues and eigenfunctions are obtained from the Monte Carlo simulation by discretizing the phase space into a collection of states. For now, the spatial extent of the system is assumed to be infinite, and the states include neutron energy intervals (or bins) and delayed neutron precursor groups. The transition rate matrix $\mathbf{Q}$ has the following form:

$$
\mathbf{Q}=\left[\begin{array}{ccc}
-q_{11} & q_{12} & \cdots \\
q_{21} & -q_{22} & \cdots \\
\vdots & \vdots & \ddots
\end{array}\right]=\left[\begin{array}{ll}
\mathbf{Q}_{n, n} & \mathbf{Q}_{n, p} \\
\mathbf{Q}_{p, n} & \mathbf{Q}_{p, p}
\end{array}\right]
$$

The quantity $q_{i i}$ is the mean net removal rate from state $i$, and $q_{i j}(i \neq j)$

is the mean rate that neutrons transition from state $i$ into $j$. Unlike typical 
continuous-time Markov processes, the rows do not sum to zero, meaning there may be an overall net gain or loss in the neutron population (as would be desired in a supercritical or subcritical system).

For these calculations, $\mathbf{Q}$ is organized into four partitions: the top-left partition describes the transition of neutrons between energy bins $\left(\mathbf{Q}_{n, n}\right)$, the top-right partition pertains to neutrons causing fission resulting in the production of delayed neutron precursors $\left(\mathbf{Q}_{n, p}\right)$, the bottom-left partition represents the emission of neutrons from precursors $\left(\mathbf{Q}_{p, n}\right)$, and the bottomright partition represents the decay (removal) of the precursors $\left(\mathbf{Q}_{p, p}\right)$. These transition rates are expressible in terms of cross sections, decay constants, and probabilities. In the top-left partition $\mathbf{Q}_{n, n}$,

$$
q_{i j}=\begin{array}{ll}
v_{i}\left(\Sigma_{R i}-\chi_{p i} \nu_{p i} \Sigma_{f i}\right), & i=j \\
v_{i}\left(\Sigma_{s i j}+\chi_{p j} \nu_{p i} \Sigma_{f i}\right), & i \neq j
\end{array},
$$

where $v_{i}, \Sigma_{R i}$, and $\Sigma_{f i}$ are the speed, removal, and fission cross sections for energy bin $i ; \nu_{p i}$ is the prompt neutrons emitted per fission for energy bin $i$; $\chi_{p i}$ is the prompt fission emission probability for energy bin $i$; and $\Sigma_{s i j}$ is the scattering cross section from energy bin $i$ to $j$. For the top-right partition $\mathrm{Q}_{n, p}$,

$$
q_{i j}=v_{i} \beta_{j} \nu_{d i} \Sigma_{f i},
$$

where $\beta_{j}$ is the delayed fraction for precursor group $j$ and $\nu_{d i}$ is the mean delayed neutron emission for energy bin $i$. For the bottom-left partition $\mathbf{Q}_{p, n}$,

$$
q_{i j}=\chi_{i j} \lambda_{i},
$$

where $\chi_{i j}$ is the emission of neutrons from precursor group $i$ into neutron energy bin $j$, and $\lambda_{i}$ is the decay constant for precursor group $i$. For the 
bottom-right partition $\mathbf{Q}_{p, p}$,

$$
q_{i j}=\begin{aligned}
& \lambda_{j}, \quad i=j \\
& 0, \quad i \neq j
\end{aligned} .
$$

These quantities are identical to the elements of the matrix form of the adjoint neutron transport equation, with the exception that the elements are actually adjoint-weighted quantities. Because the adjoint eigenvalues $\alpha^{\dagger}$ are the complex conjugates of the forward eigenvalues $\alpha$, and considering that if an eigenvalue is complex, its complex conjugate must also be an eigenvalue because the matrix is all real, the eigenvalues of the transition rate matrix are also the $\alpha$ eigenvalues of the forward neutron transport equation. The forward eigenvectors are then obtained by taking $\mathbf{Q}^{\top}$, exchanging the speeds $v_{i}$ and $v_{j}$, and finding the eigenvectors of the resultant matrix.

\subsection{Monte Carlo tallies}

The Monte Carlo simulation is carried out with a $k$-eigenvalue calculation using the power iteration technique. Technically when the system is not exactly critical, the energy spectrum is incorrect; the effect that this has on the calculated $\alpha$-eigenvalue spectrum diminishes as the number of energy bins increases and is assumed to be negligible. During the simulation, reaction rate tallies are used to estimate the $q_{i j}$ 's.

All elements are combinations of removal rates and probabilities:

$$
\begin{gathered}
\lambda_{i}=\tau_{R i}^{-1}=(\text { average decay time from precursor group } i)^{-1}, \\
v_{i} \Sigma_{R i}=\tau_{R i}^{-1}=(\text { average removal time from energy bin } i)^{-1}, \\
v_{i} \Sigma_{f i}=v_{i} \Sigma_{R i} \frac{\Sigma_{f i}}{\Sigma_{R i}}=\tau_{R i}^{-1}\left(\frac{\# \text { of fissions caused by neutrons in energy bin } i}{\# \text { of removals from energy bin } i}\right), \text { and }
\end{gathered}
$$




$$
v_{i} \Sigma_{s i j}=v_{i} \Sigma_{R i} \frac{\Sigma_{s i j}}{\Sigma_{R i}}=\tau_{R i}^{-1}\left(\frac{\# \text { of scatters from energy bin } i \text { into bin } j}{\# \text { of removals from energy bin } i}\right) .
$$

\subsection{Eigenfunction expansion coefficients}

Capturing the temporal behavior requires computing expansion coefficients $A_{n}(0)$ from the external source $S(\mathbf{r}, E, \hat{\Omega}, t)$. These are obtained from the differential equation

$$
\frac{d A_{n}(t)}{d t}=\alpha_{n} A_{n}(t)+\frac{\left\langle\psi_{n}^{\dagger}, S\right\rangle}{\left\langle\psi_{n}^{\dagger}, v^{-1} \psi_{n}\right\rangle+\sum_{m}\left\langle C_{m, n}^{\dagger}, C_{m, n}\right\rangle},
$$

where the brackets denote integration over all relevant phase space variables.

Often the source is defined as a pulse at $t=0: S(\mathbf{r}, E, \hat{\mathbf{\Omega}}, t)=S_{0}(\mathbf{r}, E, \hat{\mathbf{\Omega}}) \delta(t)$, where $\delta(t)$ is the Dirac delta function. With this source, the solution to this differential equation simplifies to

$$
A_{n}(t)=A_{n}(0) e^{\alpha_{n} t}
$$

where

$$
A_{n}(0)=\frac{\left\langle\psi_{n}^{\dagger}, S_{0}\right\rangle}{\left\langle\psi_{n}^{\dagger}, v^{-1} \psi_{n}\right\rangle+\sum_{m}\left\langle C_{m, n}^{\dagger}, C_{m, n}\right\rangle}
$$

\section{Code description and verification}

TORTE is a standalone research code written in MATLAB. It is capable of performing $k$-eigenvalue power iteration calculations with either multigroup or continuous-energy nuclear data (read from ENDF/B-VII.0 (Chadwick et al., 2006) or ENDF/B-VII.1 (Chadwick et al., 2011)). For scattering, TORTE is capable of handling both free gas and continuous $S(\alpha, \beta)$ but is 
limited in that it does not treat high-energy inelastic scattering. The aforementioned tallies for the $q_{i j}$ 's (elements of the transition rate matrix) are collected during this calculation, and the $\alpha$ eigenvalues and eigenfunctions are estimated after the transport calculation.

Verification of the multigroup tallies was completed using test problems where the multigroup cross sections are given. TORTE can reproduce the known multigroup cross sections in the transition rate matrix to within $1 \%$, which is within the statistical uncertainty of the Monte Carlo method. Verifying the continuous-energy physics is more difficult because global quantities (i.e., the calculated $k$ eigenvalue) will not match production codes because high-energy inelastic scattering is excluded. Limited verification of the continuous-energy physics was completed by tabulating free-gas scattering distributions and fission $\chi$ spectra and comparing them to known or analytic distributions. These comparisons show agreement within the statistical deviation expected from the Monte Carlo simulation.

The calculated $\alpha$-eigenvalue spectrum is verified with a highly idealized problem with a known analytic solution. ${ }^{2}$ Consider a problem where all energy groups $g$ have the same speed $v$ and downscattering may only occur into the next group. Fission is only possible in the lowest energy group $G$, which creates neutrons exclusively in the highest energy group (group 1). All cross sections in all groups are identical except for group $G$, where the fission cross section is equal to the group-to-group scattering cross section in the other groups. The eigenvalue spectrum of the operator for this problem is a 
circle in the complex plane with $G$ discrete eigenvalues given by

$$
\frac{\alpha_{n}}{v}=-\Sigma_{c}+\Sigma_{s g, g+1}\left[\nu^{1 / G} e^{2 \pi i n / G}-1\right] .
$$

Arbitrary data with $G=81\left(\Sigma_{c}=1, \Sigma_{f G}=\Sigma_{s g, g+1}=100, v=1, \nu=\right.$ $2.5)$ are used. The analytic and calculated eigenvalue spectra are given in Fig. 1. The two sets of results agree qualitatively. The analytic fundamental eigenvalue $\alpha_{0}$ is $0.13765 \mathrm{~s}^{-1}$, whereas the calculated $\alpha_{0}$ is $0.13839 \mathrm{~s}^{-1}$; these agree within $1 \%$. This small difference is due to the statistical uncertainty associated with the tally of the $q_{i j}$ 's in the transition rate matrix.

The eigenfunction expansion is tested by defining a monoenergetic highenergy $(g=1)$ pulse source, where $S_{0}(1)=5 \times 10^{4} \mathrm{~cm}^{-3} \cdot \mathrm{s}^{-1}$. Snapshots of the time evolution of the neutron flux are given in Fig. 2. At $t=0.10 \mathrm{~s}$, neutrons from the monoenergetic source begin to scatter out of energy group $g=1$ at different times, resulting in a small flux packet that begins to downscatter. At $t=0.70 \mathrm{~s}$, the flux packet continues to widen and decrease due to the different rates at which neutrons are downscattering and neutron capture. When neutrons reach energy group $g=81$, they begin to induce fission and emit neutrons in energy group $g=1$. At $t=1.60 \mathrm{~s}$, the flux packet widens to the point where it combines with the neutrons emitted from fission in energy group $g=1$. At $t=6.00 \mathrm{~s}$, individual flux packets are no longer discernible as the flux distribution begins to approach and follow the rising fundamental mode. As $t \rightarrow \infty$, the higher modes decay and the complete flux solution approaches that of the fundamental mode. For this problem, this happens

\footnotetext{
${ }^{2}$ Larsen, E.W., 2012. Personal Communication.
} 
at $t \approx 20 \mathrm{~s}$, which is long relative to the lifetime of the prompt modes. The behavior observed here is as expected for this contrived problem.

\section{Continuous-energy results}

To understand the eigenvalue spectrum with continuous-energy nuclear data, two systems are considered: one mixture that is hydrogenous and another that is anhydrogenous (graphite moderated). These are representative of highly idealized light-water and gas-cooled graphite-moderated reactors. Both are slightly supercritical systems with $G=1,000$ equal-lethargy bins ranging from energies $E_{0}=20 \mathrm{MeV}$ to $E_{G}=1 \times 10^{-11} \mathrm{MeV}$. Nuclear data are obtained from ENDF/B-VII.0, except for the continuous- $S(\alpha, \beta)$ law for graphite, which is from ENDF/B-VII.1.

\subsection{Hydrogenous mixture}

This is a delayed supercritical, continuous-energy, hydrogenous medium with a $4: 2: 1 \mathrm{H}: \mathrm{O}: \mathrm{UO}_{2}$ molecular ratio. The computed eigenvalue spectrum is given in Fig. 3, where $\alpha_{0}$ is $1.4758 \times 10^{-3} \mathrm{~s}^{-1}$.

Figure 4 shows the approximated energy spectrum at selected times for a $t=0$ pulsed neutron source in the highest energy bin. At $t=0.5 \mathrm{~ms}$, neutrons from the monoenergetic pulse source begin to downscatter. Flux depressions form at high energies as neutrons encounter resonances. Because neutrons are able to downscatter to near-zero energies in a collision with hydrogen, the neutron flux extends to lower energies. At $t=1.5 \mathrm{~ms}$, neutrons continue to downscatter as the high-energy (fast) flux finally begins to decrease. A very large flux packet forms. At $t=30.0 \mathrm{~ms}$, the fast flux continues to decrease and drops below the fundamental mode because the 
neutrons downscatter out of higher energies faster than they induce fissions that emit neutrons at higher energies. Lower energy resonances cause depressions in the flux packet as neutrons downscatter. At $t=150.0 \mathrm{~ms}$, the fast flux recovers as neutrons enter thermal energies and induce fission.

\subsection{Graphite mixture}

This is a highly scattering continuous-energy graphite medium with a 4,000 : $1 \mathrm{C}: \mathrm{UO}_{2}$ molecular ratio. It is slightly above delayed supercritical. The calculated eigenvalue spectrum is given in Fig. 5, where $\alpha_{0}$ is $1.6529 \times$ $10^{-2} \mathrm{~s}^{-1}$.

Figure 6 shows the approximated energy spectrum at selected times for a $t=0$ pulsed neutron source in the highest energy bin. At $t=0.05 \mathrm{~ms}$, neutrons from the monoenergetic pulse begin to downscatter and form a distinct flux packet because neutrons colliding with carbon cannot scatter to near-zero energies. The fast flux decreases rapidly because neutrons are not at energies at which fission can replenish the fast flux. The peaks below the lower energy boundary of the flux packet are statistical noise from the eigenfunction expansion attempting to model near-zero flux or very improbable neutrons existing at lower energies. At $t=0.50 \mathrm{~ms}$, the distinct flux packet maintains its form and continues to propagate to lower energies. The fast flux decreases considerably and forms depressions because of resonances. At $t=9.00 \mathrm{~ms}$, the flux packet encounters low energy resonances as the fast flux decreases far below the fundamental mode. Not enough neutrons are inducing fission because the majority of the neutrons are still above energies at which fission is preferential. At $t=80.00 \mathrm{~ms}$, the flux at higher energies recovers because of neutrons inducing fission. 


\subsection{Discussion}

Depressions in the neutron flux due to ${ }^{238} \mathrm{U}$ capture resonances are resolved with $G=1,000$. For the two mixtures, the depths of the resonance effects are different because of the vastly different relative concentrations of moderating nuclei. The flux packet is far more defined in Fig. 6 because the neutrons lose only a fraction of their energy in a collision with a carbon nucleus. This also contributes to the fast flux decreasing below the fundamental mode more than the fast flux of the hydrogenous mixture. Furthermore, the fast flux in the graphite mixture tends to oscillate more, rising and decreasing before approaching the fundamental mode solution. This is a result of more complex eigenvalues with larger real components (relative to the fundamental) in the eigenvalue spectrum of the graphite-moderated mixture than in that of the hydrogenous mixture. The fast flux in the fundamental mode is greater in the hydrogenous mixture because it has a much lower concentration of scattering nuclei, which significantly hardens the energy spectrum.

These problems show the potential for the use of eigenfunction expansion to approximate the time dependence of the energy spectrum. In both problems, the higher kinetic modes do not decay to the fundamental mode solution for a few seconds. During these first few seconds (and particularly the first fraction of a second), the fundamental mode solution is a completely inaccurate representation of the energy-dependent flux shape. In addition, recent comparisons to true time-dependent Monte Carlo solutions (using the same code physics) show the accuracy of the eigenfunction expansion in approximating the time-dependent behavior of the flux. 


\section{Conclusions and future work}

The transition rate matrix method, the continuous-time analog of the fission matrix method, can accurately compute $\alpha$ eigenvalues and eigenvectors of infinite media. These can be applied to model transient behavior of the energy spectrum. The standalone research code TORTE is capable of performing these calculations with either multigroup or continuous-energy nuclear data. The ability of TORTE to compute multigroup $\alpha$-eigenvalue spectra has been verified, as has much of its continuous-energy scattering physics. TORTE is shown to be able to calculate continuous-energy $\alpha$-eigenvalue spectra, and results have been obtained for both hydrogenous and anhydrogenous mixtures.

Future work will focus on studying the numerical convergence of the $\alpha$ eigenvalues and quantifying their uncertainties. Research will also be performed to extend this method to calculate spatial modes in multiple dimensions in addition to just energy modes.

\section{Acknowledgements}

Funding for this work was provided by the US Department of Energy National Nuclear Security Administration Advanced Scientific Computing and Nuclear Criticality Safety programs.

\section{References}

\section{References}

S. E. Carney, F. B. Brown, B. C. Kiedrowski, W. R. Martin, Fission Matrix Capability for MCNP Monte Carlo, in: Trans. Am. Nucl. Soc., vol. 107, 
2012 .

G. R. Grimmett, D. R. Stirzaker, Sec. 6.9: Continuous-time Markov chains, in: Probability and Random Processes, 3rd Ed., Oxford University Press, Oxford, UK, 2001.

T. R. Hill, Efficient Methods for Time Absorption $(\alpha)$ Eigenvalue Calculations, Tech. Rep. LA-9602-MS, Los Alamos National Laboratory, 1983.

T. Yamamoto, Higher Order $\alpha$ Mode Eigenvalue Calculation by Monte Carlo Power Iteration, Progress in Nuclear Science and Technology 2 (2011) 826835.

MATLAB, version 7.10.0, The Mathworks Inc., Natick, Massachusetts, 2010.

E. W. Larsen, P. F. Zweifel, On the Spectrum of the Linear Transport Operator, Journal of Mathematical Physics 15 (1974) 1987-1997.

M. B. Chadwick, P. Obložinký, M. Herman, et al., ENDF/B-VII.0: Next Generation Evaluated Nuclear Data Library for Nuclear Science and Technology, Nucl. Data Sheets 107 (2006) 2931-3060.

M. B. Chadwick, P. Obložinký, M. Herman, et al., ENDF/B-VII.1: Nuclear Data For Science and Technology: Cross Sections, Covariance, Fission Product Yields and Decay Data, Nucl. Data Sheets 112 (2011) 2887-2996. 


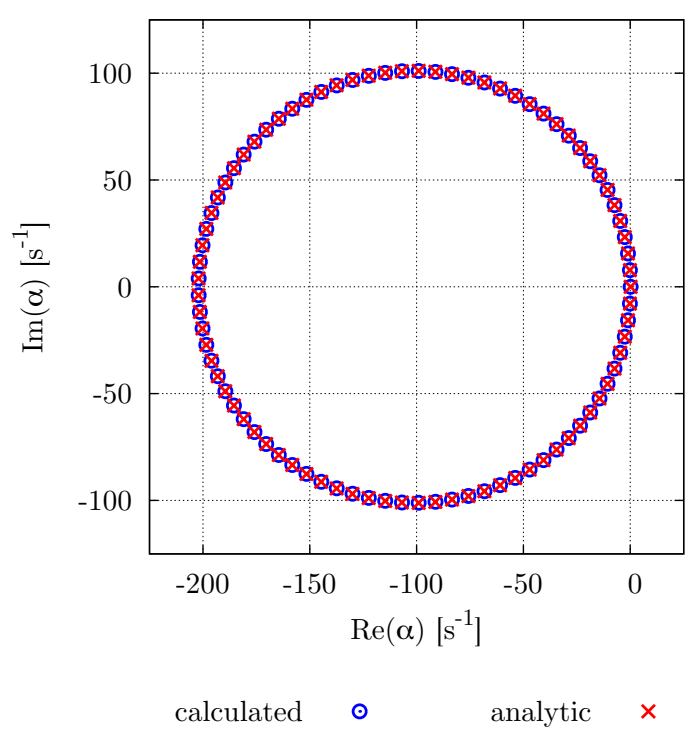

Figure 1: The $\alpha$-eigenvalue spectrum for the 81-group verification problem. 

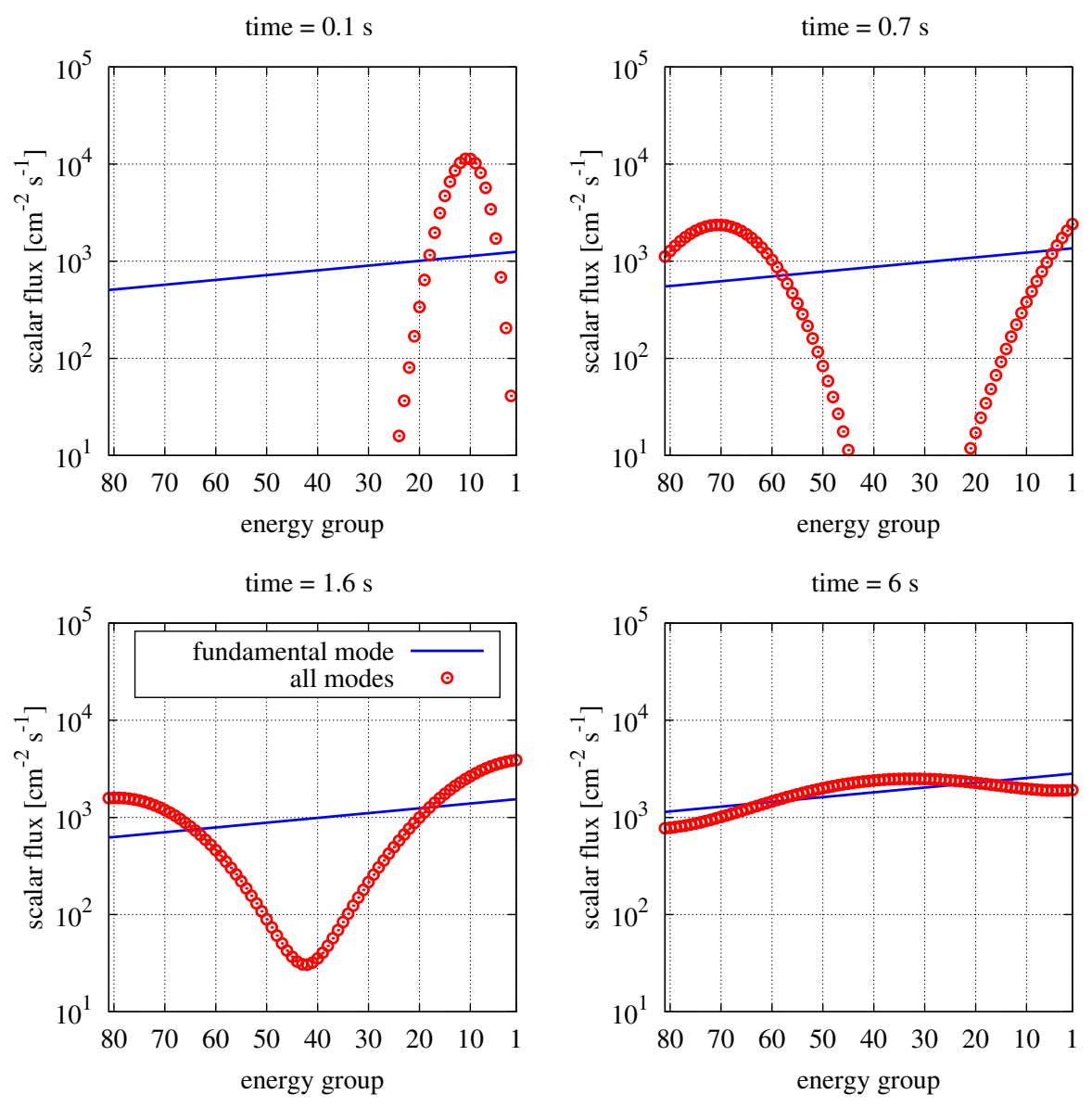

Figure 2: Snapshots of a transient for the 81-group verification problem (legend applies to all snapshots). 


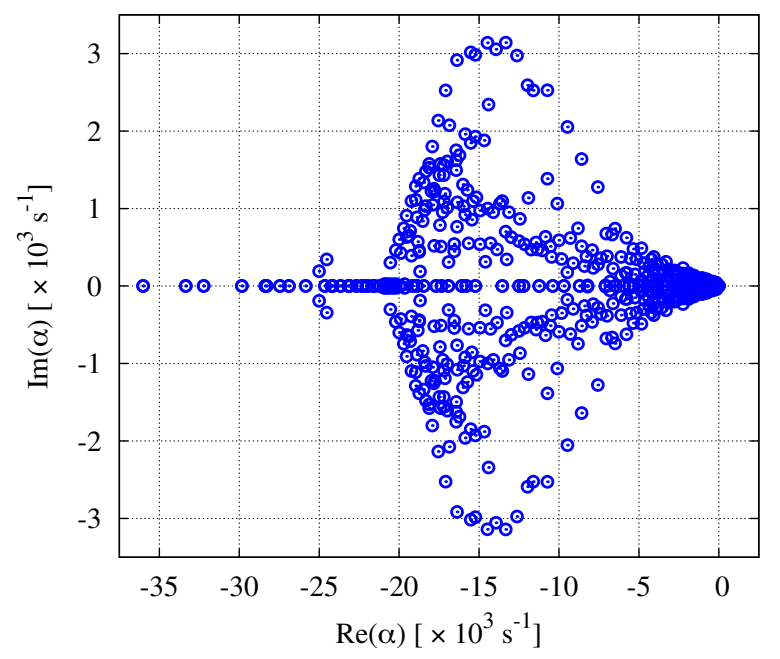

Figure 3: $\alpha$-eigenvalue spectra for a hydrogenous mixture. 

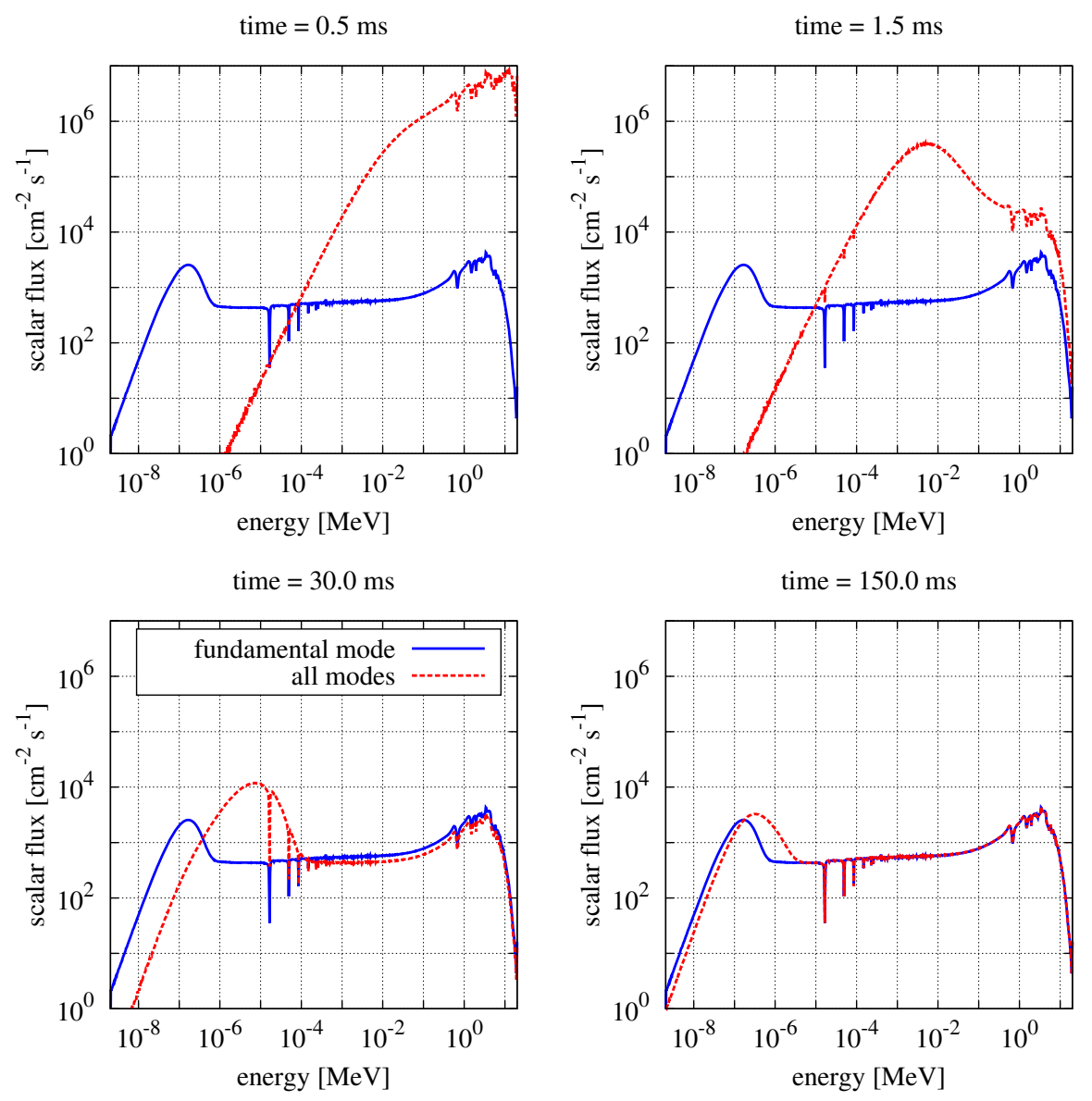

Figure 4: Snapshots of a transient in the hydrogenous mixture (legend applies to all snapshots). 


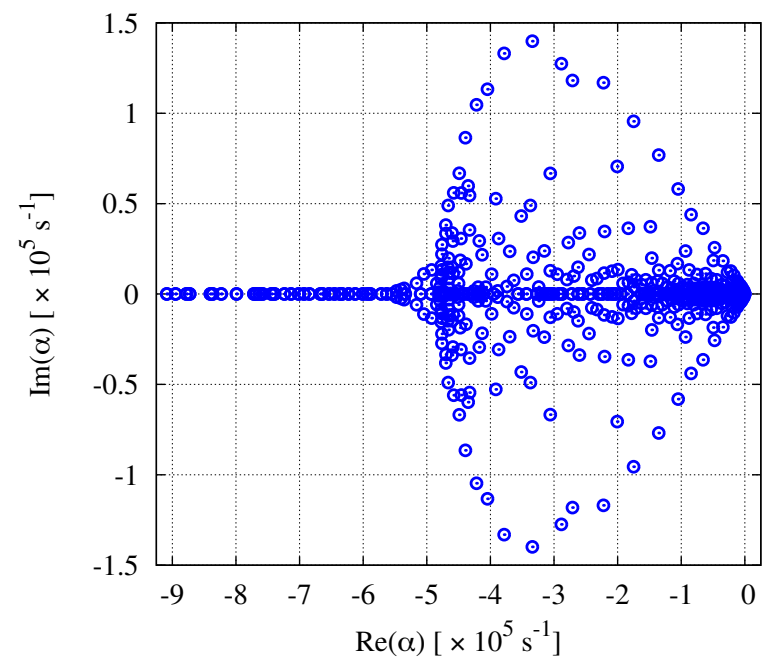

Figure 5: $\alpha$-eigenvalue spectra for a graphite-moderated mixture. 

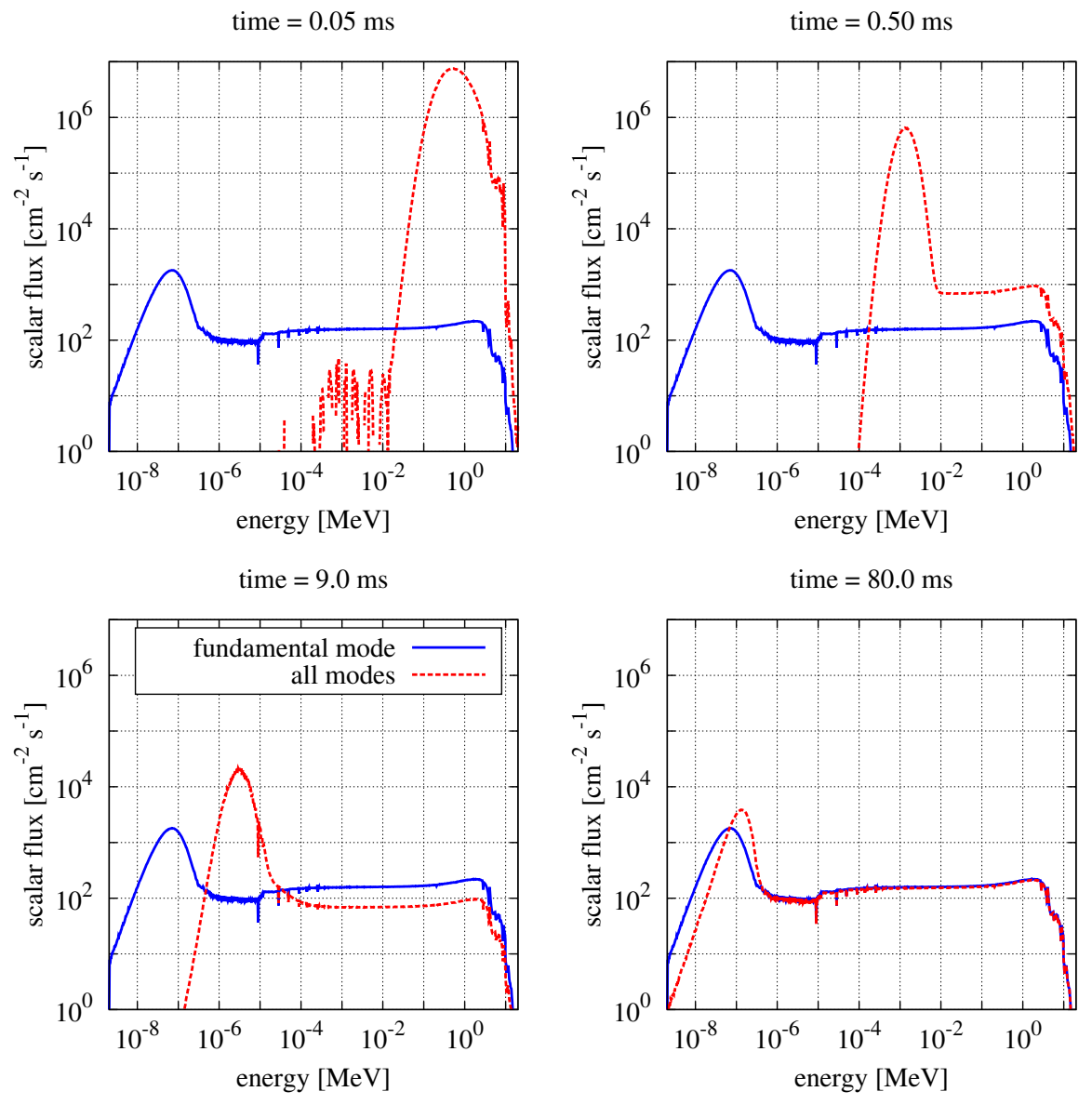

Figure 6: Snapshots of a transient in the graphite mixture (legend applies to all snapshots). 